\title{
Aorto-Esophageal Fistula After Thoracic Endovascular Aortic Repair: Successful Open Treatment
}

\author{
Julia Dumfarth, $\mathrm{MD}^{1 *}$, Hannes Dejaco, $\mathrm{MD}^{2}$, Christoph Krapf, MD ${ }^{1}$, Thomas Schachner, $\mathrm{MD}^{1}$, \\ Heinz Wykypiel, $\mathrm{MD}^{3}$, Thomas Schmid, $\mathrm{MD}^{3}$, Johann Pratschke, MD, $\mathrm{PhD}^{3}$, Michael Grimm, $\mathrm{MD}^{1}$ \\ ${ }^{1}$ Department of Cardiac Surgery, University Clinic Innsbruck, Innsbruck, Austria; ${ }^{2}$ Department of Anesthesiology, University Clinic Innsbruck, \\ Innsbruck, Austria; and ${ }^{3}$ Department of Visceral, Transplant and Thoracic Surgery, University Clinic Innsbruck, Innsbruck, Austria
}

\begin{abstract}
We present the case of a 56 -year-old patient suffering from an aorto-esophageal fistula after complex treatment of acute Type A dissection including thoracic endovascular aortic repair (TEVAR) of the descending aorta. Open surgical descending replacement using a pericardial patch, as well as esophagectomy, was performed. After a long and complicated hospital stay, the patient finally recovered and was discharged in stable condition. By choosing an aggressive surgical approach the patient survived this devastating complication of TEVAR, which is associated with high mortality.

Copyright $\odot 2013$ Science International Corp.
\end{abstract}

\section{Key Words}

Secondary surgical procedure - TEVAR - Aorto-esophageal fistula $\cdot$ Case description

\section{Introduction}

We present the case of a 56-year-old patient who underwent replacement of the ascending aorta and aortic arch due to acute Type A dissection at an external department. The patient suffered from arterial hypertension and unilateral paresis of the recurrent laryngeal nerve after the primary surgery.

During follow-up, the patient showed a progressive aneurysm of the descending aorta and was therefore treated with subclavian transposition

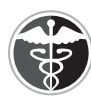

Fax +1 2037853346

E-Mail: aorta@scienceinternational.org

http://aorta.scienceinternational.org (c) 2014 Aorta.

Published by Science International Corp. ISSN 2325-4637

Accessible online at:

http://aorta.scienceinternational.org in combination with thoracic endovascular aortic repair (TEVAR) (Medtronic Valiant, $42 \times 100$, Medtronic Vascular, Santa Rosa, CA) 8 months after emergency surgery. Two months after TEVAR the patient was readmitted with hematemesis and significant blood loss. Computer tomography (CT) scan revealed contained rupture of the descending aorta distal to the stent graft. The patient then again underwent TEVAR (Medtronic Valiant, $42 \times 140$, Medtronic Vascular, Santa Rosa, CA) for distal elongation of the stent graft in an emergency setting. Gastroscopy revealed erosion of the esophagus, which was treated with an esophageal stent placement. Ten days after TEVAR and esophageal stent placement, control gastroscopy was performed and the patient was discharged home under stable conditions.

One month after the second intervention the patient was scheduled for removal of the esophageal stent. Gastroscopy after removal of the stent revealed a large defect in the esophagus with an aortoesophageal fistula, covering approximately a third of the esophageal circumference (Fig. 1). Based on these findings, a CT scan was performed, which confirmed the diagnosis of an aorto-esophageal fistula with signs of mediastinitis (Fig. 1).

At this time point the patient was stable and was transferred to our center for surgical repair. Preopera-

*Corresponding author:

Julia Dumfarth, MD

Department of Cardiac Surgery, University Clinic Innsbruck

Anichstraße 35

6020 Innsbruck, Austria

Tel: +1 43512504 0, Fax: +1 43512504 22528, E-Mail: Julia.Dumfarth@i-med.ac.at 


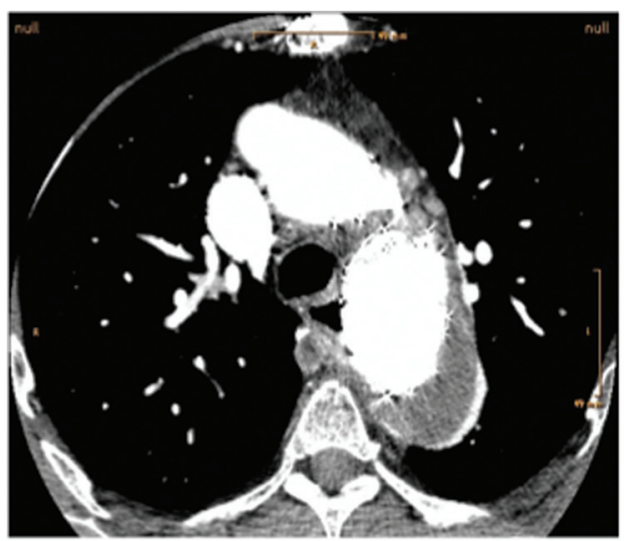

A

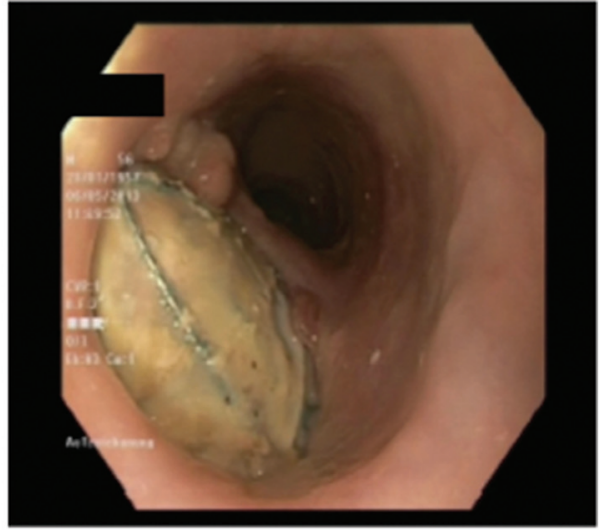

B

Figure 1. (A) CT scan revealing migration of the stent graft into the esophageal wall. (B) Gastroscopic finding showing a big defect of the esophageal wall and presence of an aorto-esophageal fistula.

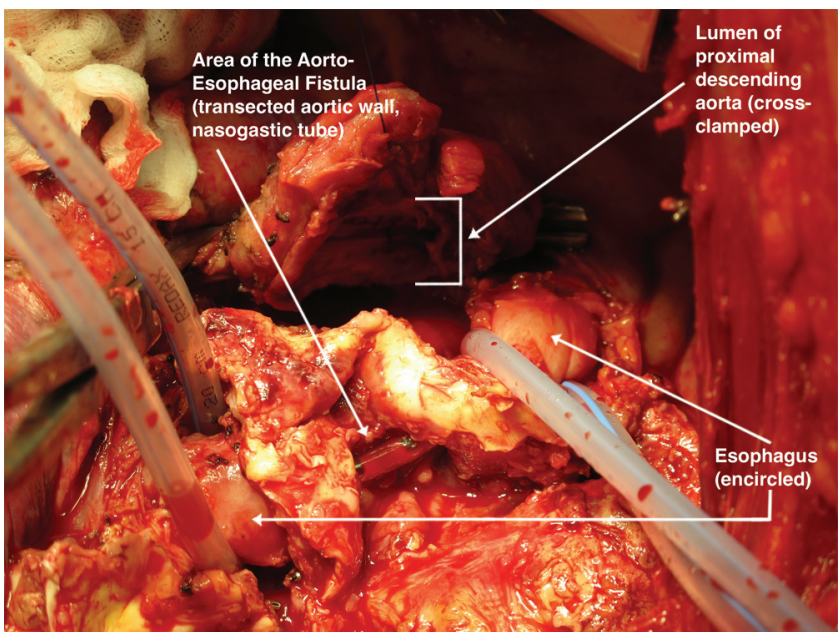

Figure 2. Intraoperative view of defect in the esophagus and aortic wall.

tively, a percutaneous endoscopic gastrostomy (PEG) tube was placed due to the planned surgical esophagectomy. Through left lateral thoracotomy the aortoesophageal fistula was identified (Fig. 2). The patient was placed on femoral-femoral bypass through the groin vessels. The descending aorta was clamped distal to the aortic arch and proximal to the diaphragm. As a first step, evaluation of the defect of the esophageal wall was performed. As the fistula covered nearly half of the esophageal circumference, resection of about $8 \mathrm{~cm}$ of the esophagus was performed with a stapling method (Endo-GIA, blue cartridge, Covidien Intl., Mansfield, MA). The descending aorta was transected and the two stent grafts could successfully be removed. Due to infection risk of the surrounding tissue, a biological replacement

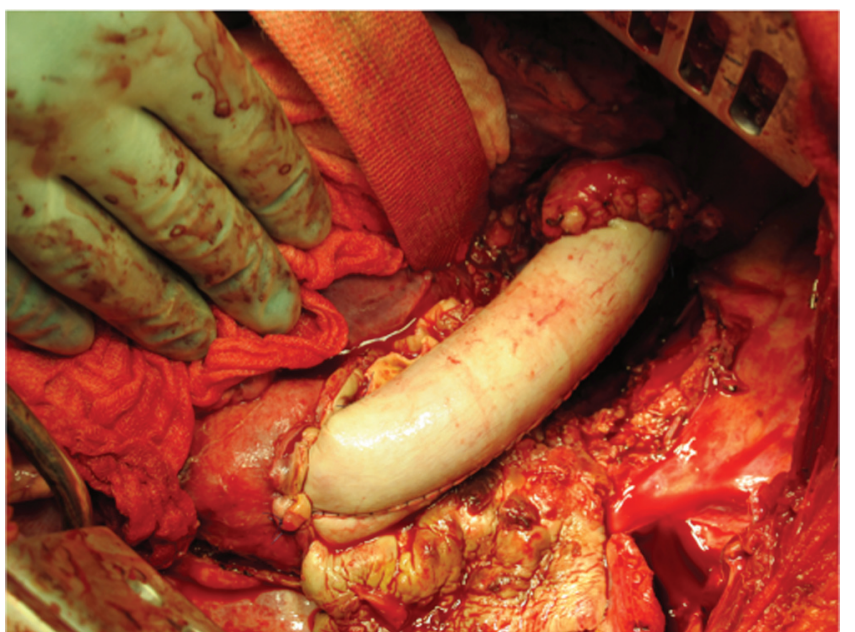

Figure 3. Descending aortic replacement using a pericardial tube.

of the descending aorta was performed using a pericardial patch (Fig. 3). The aortic graft was de-aired and the aortic cross-clamp was removed after 124 minutes. The patient was weaned from bypass after 140 minutes. After successful partial esophagectomy and descending aortic replacement, a cervical salivary fistula (T-Tube, 12F) was created via a left cervical approach and the patient was taken to the intensive care unit (ICU).

The patient had a complicated and long postoperative recovery at the ICU, requiring reintubation and tracheotomy on the 12th postoperative day. He suffered from two episodes of pneumonia presumably due to aspiration and received extensive antibiotic treatment. Three months after esophagectomy, a reconstruction of the esophagus was 
performed by gastric tube pull up with a stapled (CEEA, blue load, Covidien, Intl., Mansfield, MA) endto-side esophagogastrostomy, a pyloromyotomy, and a jejunal catheter placement.

Nearly 4 months after descending aortic replacement, the patient recovered completely without wound healing disorders. He was in a stable respiratory state and could be discharged without neurologic impairment and in stable condition.

The patient was seen in outpatient clinic for a follow-up visit 6 months after descending replacement. He was still in recovery with complaints about pain along the thoracotomy wound. Furthermore, the patient was still in a cachectic nutritional status.

\section{Discussion}

Secondary surgical interventions after TEVAR are needed in 2.2-7.4\% of all patients [1,2]. While overall mortality in those challenging cases ranges from 11.5 to $19 \%$, survival of patients undergoing surgery due to aorto-esophageal fistula is still very poor [2-6]. Muradi et al. [7] summarized published cases on secondary aortoesophageal fistula after TEVAR and reported a mortality of $75 \%$. Analysis of 36 patients in the European registry of endovascular aortic repair also pointed out the high mortality of this devastating complication and the best 1 -year survival rate in patients undergoing aggressive surgical treatment [6].

The mechanisms of aorto-esophageal fistula might consist of a combination of stent graft coverage of the arteries in the midesophageal segment causing ischemic necrosis or, in cases of contained rupture, possible development of a resorption hematoma leading to wall fatigue of the esophagus [1,2]. Further hypotheses on the pathomechanism include direct erosion of the relatively rigid stent graft through the aortic wall into the esophagus, pressure necrosis of the esophageal wall due to the continuing forces of self-expanding endoprosthesis, and infection of stent graft prosthesis leading to erosion of the esophageal wall [5]. Opening the mediastinal pleura via lateral thoracotomy and decompressing the posterior mediastinum could emerge as a preventive treatment [2].

In this case, development of the aorto-esophageal fistula was most probably based on a combination of different mechanisms. Erosion of the esophagus occurred probably due to ischemia induced by a resorption hematoma of the descending aorta in the first instance. By placing an esophageal stent, malperfusion of the esophageal wall was aggravated and finally resulted in a pressure necrosis induced by the forces of two self-expanding devices. The patient was initially treated at an external department and was transferred for treatment of the aorto-esophageal fistula to our department. Occurrence of esophageal erosion after TEVAR was probably the first sign of malperfusion and impending fistulation. In accordance with published data, the esophageal stenting-only approach is associated with poor 1 -year survival of only $17 \%$ [6]. After confirmation of aorto-esophageal fistula, aggressive surgical treatment was chosen, which finally resulted in a prolonged and complicated hospital stay and survival of the patient.

Similar cases are described in the literature using either a bovine pericardial patch or other material for descending replacement, such as silver-coated polyester grafts or autografts $[3,4,6]$. All these materials aim to avoid Dacron grafts, which might be at higher risk for reinfection. Removing all infected material, performing extensive debridement, and using a selfmade pericardial tube as neoarta provides excellent and durable results [8].

\section{Conflict of Interest}

The authors have no conflict of interest relevant to this publication.

\section{Comment on this Article or Ask a Question}

\section{References}

1. Girdauskas $E$, Falk V, Kuntze $T$, Borger MA, Schmidt A, Scheinert $D$, et al. Secondary surgical procedures after endovascular stent grafting of the thoracic aorta: successful approaches to a challenging clinical problem. $J$ Thorac Cardiovas Surg. 2008;136:1289-1294. 10.1016/j.jtcvs.2008.05.053
2. Dumfarth J, Michel M, Schmidli J, Sodeck G, Ehrlich M, Grimm M, et al. Mechanisms of failure and outcome of secondary surgical interventions after thoracic endovascular aortic repair (TEVAR). Ann Thorac Surg. 2011;91:1141-1146. 10.1016/j.athoracsur. 2010.12.033
3. Canaud L, Alric P, Gandet T, Ozdemir BA, Albat B, Marty-Ane C. Open surgical secondary procedures after thoracic endovascular aortic repair. Eur J Vasc Endovasc Surg. 2013; 45:667-674. 10.1016/j.ejvs.2013.08.022

4. Kieffer E, Chiche L, Gomes D. Aortoesophageal fistula: value of in situ aortic allograft 
replacement. Ann Surg. 2003;238:283-290. 10. 1097/01.sla.0000080828.37493.e0

5. Eggebrecht H, Mehta RH, Dechene A, Tsagakis $K$, Kühl H, Huptas S, et al. Aortoesophageal fistula after thoracic aortic stent-graft placement: a rare but catastrophic complication of a novel emerging technique. J Am Coll Cardiol Cardiovasc Interv. 2009;2:570-576. 10.1016/j.jcin.2009.03.010

6. Czerny M, Eggebrecht H, Sodeck G, Weigang $E$, Livi $U$, Verzini $F$, et al. New insights regarding the incidence, presentation and treatment options of aortooesophageal fistulation after thoracic endovascular aortic repair: the European Registry of Endovascular Aortic Repair Complications. Eur J Cardiothorac Surg. 2013; in press. 10.1093/ejcts/ezt393

7. Muradi A, Yamaguchi M, Kitagawa A, Nomura Y, Okada T, Okita $Y$, et al. Secondary aortoesophageal fistula after thoracic endovascular aortic repair for a huge aneurysm. Diagn Interv Radiol. 2013;19:81-84. 10.4261/13053825.DIR.5912-12.1

8. Czerny $M$, von Allmen R, Opfermann $P$, Sodeck G, Dick F, Stellmes A, et al. Self-made pericardial tube graft: a new surgical concept for treatment of graft infections after thoracic and abdominal aortic procedures.
Ann Thorac Surg. 2011;92:1657-1662. 10. 1016/j.athoracsur.2011.06.073

Cite this article as: Dumfarth J, Dejaco H, Krapf C, Schachner T, Wykypiel H, Schmid T, Pratschke J, Grimm M. AortoEsophageal Fistula After Thoracic Endovascular Aortic Repair: Successful Open Treatment.Aorta2014;2(1):37-40. DOl:http:// dx.doi.org/10.12945/j.aorta.2014.13-056 